\title{
Eficiência dos testes COPAN (Microplate e Single) na detecção de resíduos de antimicrobianos no leite
}

\author{
[Efficacy of COPAN (Microplate and Single kits) in detection of antimicrobials residues in milk] \\ C.G.M.S.C. Tenório ${ }^{1}$, M.M.O.P. Cerqueira ${ }^{2}$, R.P. Viegas ${ }^{1}$, M.F.S. Resende ${ }^{1}$, \\ D.L. Clinquart ${ }^{3}$, A.K.R. Santos ${ }^{1}$, M.R. Souza ${ }^{2}$, C.F.A.M. Penna ${ }^{2}$ \\ ${ }^{1}$ Alunas de pós-graduação - EV-UFMG - Belo Horizonte, MG \\ ${ }^{2}$ Escola de Veterinária - UFMG - Belo Horizonte, MG \\ ${ }^{3}$ Aluno de graduação - EV-UFMG - Belo Horizonte, MG
}

\begin{abstract}
RESUMO
Avaliou-se a eficiência dos testes microbiológicos COPAN (Microplate e Single) para detecção de resíduos de 13 antimicrobianos inoculados em leite isento de resíduos. Foram utilizadas quatro concentrações, sendo a primeira equivalente à metade do limite de detecção declarado pelo fabricante (C1); a segunda equivalente ao limite máximo de resíduos (LMR) estabelecido pela legislação brasileira (C2); a terceira equivalente ao limite de detecção declarado pelo fabricante (C3); e a quarta equivalente ao dobro do limite de detecção declarado pelo fabricante (C4). Os testes apresentaram 100\% de sensibilidade na detecção de amoxicilina, ampicilina, cloxacilina, penicilina, tilosina, sulfadiazina, sulfametoxazol, e 96,7\% na detecção de oxacilina para as concentrações informadas pelo fabricante como limite de detecção. Em relação às concentrações estabelecidas pela legislação brasileira, verificou-se que os testes foram capazes de detectá-las para a maioria dos antimicrobianos avaliados. Os testes não se mostraram eficientes na detecção das concentrações estabelecidas pela legislação para eritromicina, oxitetraciclina, tetraciclina, trimetoprim e gentamicina (Microplate).
\end{abstract}

Palavras-chave: leite, antimicrobianos, resíduos, teste microbiológico

\begin{abstract}
The efficacy of the microbiological kits COPAN (Microplate and Single) for detection of antimicrobials residues was tested. Thirteen drugs were inoculated into milk without residues at four concentration levels: $C 1$ - half of the detection threshold declared by the manufacturer, C2 - the maximum residues threshold established by the Brazilian legislation, $\mathrm{C} 3$ - the detection threshold declared by the manufacturer, and C4 two fold the detection threshold declared by the manufacturer. The tests showed $100 \%$ efficient in detection of amoxicillin, cloxacillin, penicillin, tilosin, sulfadiazine, and sulfa methoxazol; and 96.7\% for oxacillin considering the concentration stated by the manufacturer as threshold level. Regarding the concentrations established by the Brazilian legislation, all tested kits were able to detect most of the evaluated antimicrobials. The kits were not efficient in detection of the concentration levels established by the Brazilian legislation for erythromycin, oxytetracyclin, tetracyclin, trimetoprim, and gentamycin (Microplate).
\end{abstract}

Keywords: milk, antimicrobials, residues, microbiological test

\section{INTRODUÇÃO}

A presença de resíduos de antimicrobianos no leite de consumo é preocupante por representar riscos à saúde do consumidor e por interferir na produção dos derivados (Costa, 1996; Brito, 2003; Nero et al., 2004).

Recebido em 1 de agosto de 2008

Aceito em 4 de fevereiro de 2009

E-mail: claricegebara@yahoo.com.br
Segundo o Codex Alimentarius (Instrução..., 1999), o resíduo de uma droga veterinária é a fração da droga, de seus metabólitos, produtos de conversão ou reação, e impurezas que permanecem no alimento originário de animais tratados com tais drogas.

Os antimicrobianos podem ser administrados aos animais pelas vias intramuscular, intravenosa, 
subcutânea, oral, tópica, intramamária ou intrauterina, sendo utilizados no tratamento de doenças infecciosas de vacas leiteiras, principalmente da mastite, e podem gerar resíduos no leite (Brady e Katz, 1988; Mitchel et al., 1998) por períodos variáveis. Esses resíduos podem interferir nos processamentos tecnológicos de fabricação de alimentos fermentados, como os queijos, ocasionando perdas econômicas.

Segundo o Ministério da Agricultura, Pecuária e Abastecimento - MAPA (Portaria..., 2002), não é permitido o envio de leite a estabelecimento industrial quando oriundo de animais que estejam sendo submetidos a tratamento com medicamentos de uso veterinário em geral, passíveis de eliminação pelo leite. Por esse motivo, o leite desses animais deve ser separado pelo período recomendado pelo fabricante, de forma a assegurar que os resíduos da droga não sejam superiores aos níveis fixados em normas específicas.

Os antimicrobianos possuem grande variação em relação à sua toxicidade (Instrução..., 1999), sendo o risco à saúde do consumidor representado por reações alérgicas, que podem, até mesmo, desencadear choque anafilático em indivíduos particularmente sensíveis (Brady e Katz, 1988; Johnsson, 1993; Costa, 1996; Brito, 2006). Segundo Moreira e Morais (2002), baixos níveis de antimicrobianos administrados aos animais podem selecionar in vivo bactérias multirresistentes. Os resíduos de antimicrobianos possibilitam a transferência de resistência múltipla entre os microrganismos através de plasmídios ou de elementos genéticos móveis (Schliephake, 1998; Borges et al., 2000; Carneiro et al., 2007). Segundo Machado et al. (2008), cepas de Staphylococci coagulase-negativas, isoladas no Brasil, apresentaram alto grau de resistência a antimicrobianos, podendo ser consequência do uso intensivo dessas drogas.

Devido a todos esses fatores, é recomendada a frequente monitorização da presença de resíduos de antimicrobianos em leite, sendo adotados como referência os valores estabelecidos pela legislação brasileira e internacional. $O$ leite contendo substâncias inibitórias acima dos limites legais é considerado inaceitável para utilização, podendo resultar em perda total para os produtores. Consequentemente, a disponibilidade de testes sensíveis, rápidos e baratos para a medição de resíduos de antimicrobianos no leite é uma necessidade (Ramírez et al., 2001).

Atualmente, existem vários kits comerciais para pesquisa de resíduos de antimicrobianos em leite, disponíveis no mercado, de fácil realização, sem a necessidade de instrumentos sofisticados e pessoal treinado, sendo esses kits relativamente baratos e mecanizados, possibilitando análise de várias amostras ao mesmo tempo. Entre eles estão os que se baseiam na inibição do crescimento microbiano como BR-Test (Brilliant Black Reduction Test), nas versões BR-Test Blue Star e BR-Test AS $^{1}$, Charm Farm Test e Charm inibition assay $^{2}$, Delvotest-P e Delvotest$\mathrm{SP}^{3}$ e Copan ${ }^{\circledR}$ ATK P \& S Microplate e Single ${ }^{4}$ (Costa, 1996; Cerqueira, 2003).

Há também os testes que se baseiam em uma afinidade específica que os antimicrobianos têm com certos sítios de receptores na parede celular de microrganismos. São exemplos deste tipo de teste: Charm Cowside Test, Charm I Test e Charm II Test ${ }^{2}$. Alguns testes utilizam a técnica conhecida como ELISA (Enzyme Linked Immunosorbent Assay) para a detecção de resíduos de antimicrobianos no leite, como: CITE Probe ${ }^{\circledR}$ (para gentamicina e tetraciclinas) e CITE ${ }^{\circ}$ Sulfa-trio (para sulfametazina, sulfatiazol, sulfametazina) ${ }^{5}$; Lactek (para betalactâmicos, sulfametazina e gentamicina $)^{1}$; Penzyme ${ }^{\circledR}{ }^{6}$, Snap TM (para betalactâmicos e

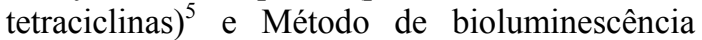
$(\mathrm{ATP})^{7}$ (Costa, 1996; Cerqueira, 2003).

No Brasil, ainda existem poucos trabalhos realizados comparando os diferentes métodos existentes no mercado. Isto dificulta a avaliação dos kits e a adoção de um método padrão. Muitos são ainda novos no mercado e necessitam de avaliações para sua validação no país (Hotta, 2003).

Um pré-requisito essencial para programas de avaliação de resíduos de antimicrobianos no leite é a disponibilidade de testes analíticos precisos e

\footnotetext{
${ }^{1}$ Idetek Inc. - EUA.

${ }^{2}$ Charm Sciences Inc. - EUA.

${ }^{3}$ Gist-Brocades Food Ingredients Inc. - EUA.

${ }^{4}$ Copan - Itália.

${ }^{5}$ IDEXX Laboratories Inc. - EUA.

${ }^{6}$ UCB-Bioproducts S.A. - Belgica.

${ }^{7}$ Biosys S.A. - France.
} 
efetivos. Na prática, há necessidade de um conjunto de testes analíticos que sejam muito rápidos (testes de triagem) para avaliação do leite antes de ser descarregado na indústria, e outros, extremamente precisos, para avaliar e confirmar resultados de testes de triagem. Para triagem, são comumente empregados os testes microbiológicos e testes específicos para antimicrobianos individuais ou grupos de antimicrobianos (Brito, 2003).

Este trabalho teve o objetivo de avaliar a eficiência de dois testes microbiológicos (COPAN ATK P \& $\mathrm{S}$ Microplate e Single) para pesquisa de resíduos de diferentes antimicrobianos em leite cru.

\section{MATERIAL E MÉTODOS}

Soluções-padrão (em solvente compatível) com 13 diferentes antimicrobianos foram adicionadas em amostras de leite (isento de resíduos) em diferentes concentrações. Foram utilizados antimicrobianos dos grupos: betalactâmicos: amoxicilina, ampicilina, cloxacilina, oxacilina e penicilina $\mathrm{G}$; tetraciclinas: oxitetraciclina e tetraciclina; macrolídeos: eritromicina e tilosina; sulfonamidas: sulfadiazina e sulfametoxazol; aminoglicosídeos: gentamicina; vários: trimetoprim. As concentrações de adição foram estabelecidas considerando-se o limite de detecção declarado pelo fabricante e o limite máximo de resíduo (LMR) estabelecido pela legislação brasileira (Instrução..., 1999) (Tab. 1), sendo a primeira equivalente à metade do limite de detecção declarado pelo fabricante $(\mathrm{C} 1)$; a segunda, ao limite máximo de resíduos (LMR) estabelecido pela legislação brasileira (C2); a terceira, ao limite de detecção declarado pelo fabricante $(\mathrm{C} 3)$ e a quarta, ao dobro do limite de detecção declarado pelo fabricante $(\mathrm{C} 4)$.

Para a realização do teste na versão Microplate, $100 \mu \mathrm{L}$ de leite contendo as concentrações de antimicrobianos descritas na Tab. 1 foram transferidos para cada orifício da placa-teste, sendo todas as concentrações repetidas 30 vezes. Para a versão Single, foram realizadas oito repetições de cada concentração. Também foram utilizadas amostras para controle negativo (leite em pó desnatado isento de resíduos ${ }^{8}$, reconstituído com água estéril) e controle positivo (leite adicionado de concentração significativa de antimicrobiano). Após a inoculação do leite no kit, este foi incubado em banho-maria a $64^{\circ} \mathrm{C} \pm 1^{\circ} \mathrm{C}$ por três horas. A leitura foi feita observando-se a presença ou não de alteração na coloração do kit (violeta $=$ positivo, amarelo= negativo), ressaltando que coloração entre o violeta e o amarelo (suspeito) é considerada positiva, pois essa coloração indica que há presença de inibidores, porém essa concentração existente está próxima à de detecção do teste.

Os procedimentos de avaliação dos kits COPAN seguiram as recomendações prescritas no Guia para validação de métodos EURACHEM (Eurachem ..., 1998), sendo realizados sob condições assépticas.

Tabela 1. Concentrações dos antimicrobianos adicionados ao leite, testadas pelos kits Microplate (M) e Single $(\mathrm{S})$

\begin{tabular}{lcccccccc}
\hline & \multicolumn{9}{c}{ Concentração $(\mu \mathrm{g} / \mathrm{L})$} \\
\cline { 2 - 10 } Antimicrobiano & \multicolumn{2}{c}{$\mathrm{C} 1$} & \multicolumn{1}{c}{$\mathrm{C} 2$} & $\mathrm{C} 3$ & \multicolumn{2}{c}{$\mathrm{C} 4$} \\
\cline { 2 - 10 } & $\mathrm{M}$ & $\mathrm{S}$ & $\mathrm{M}$ & $\mathrm{S}$ & $\mathrm{M}$ & $\mathrm{S}$ & $\mathrm{M}$ & $\mathrm{S}$ \\
\hline Amoxicilina cristalina & 1,75 & 2,0 & 4,0 & 4,0 & 3,5 & 4,0 & 7,0 & 8,0 \\
Ampicilina anidra cristalina & 2,0 & 2,0 & 4,0 & 4,0 & 4,0 & 4,0 & 8,0 & 8,0 \\
Cloxacilina sódica mono-hidratada & 12,5 & 12,5 & 30 & 30 & 25 & 25 & 50 & 50 \\
Oxacilina sódica mono-hidratada & 7,5 & 7,5 & 30 & 30 & 15 & 15 & 30 & 30 \\
Penicilina G sódica & 1,25 & 1,25 & 4,0 & 4,0 & 2,5 & 2,5 & 5,0 & 5,0 \\
Cloridrato de Tetraciclina & 50 & 50 & 100 & 100 & 100 & 100 & 200 & 200 \\
Oxitetraciclina di-hidratada Sigmaultra & 75 & 75 & 100 & 100 & 150 & 150 & 300 & 300 \\
Estearato de Eritromicina & 150 & 100 & 40 & 40 & 300 & 200 & 600 & 400 \\
Tartarato de Tilosina & 37,5 & 50 & - & - & 75 & 100 & 150 & 200 \\
Sulfadiazina & 25 & 25 & 100 & 100 & 50 & 50 & 100 & 100 \\
Sulfametoxazol & - & - & 100 & 100 & - & - & - & - \\
Sulfato de Gentamicina & 100 & 125 & 200 & 200 & 200 & 250 & 400 & 500 \\
Trimetoprim & 100 & 100 & 50 & 50 & 200 & 200 & 400 & 400 \\
\hline
\end{tabular}

C 1: metade do limite de detecção declarado pelo fabricante; C 2: limite máximo de resíduos (IN 42, OMS e Codex Alimentarius); C 3: limite de detecção declarado pelo fabricante; C 4: o dobro do limite de detecção declarado pelo fabricante.

${ }^{8}$ Difco - EUA. 


\section{RESULTADOS E DISCUSSÃO}

Os kits para detecção de resíduos de antimicrobianos COPAN, Microplate e Single, foram avaliados quanto ao seu limite de detecção. Em relação às concentrações de detecção testadas, foi verificado que os testes foram capazes de detectar a presença dos antimicrobianos nas concentrações declaradas pelo fabricante (C3). Para alguns antimicrobianos, os testes também detectaram a C2 (LMR) (Tab. 2 e 3).

Tabela 2. Detecção (\%) de diferentes concentrações (C) de antimicrobianos adicionados em leite pelo teste Microplate (COPAN, Itália)

\begin{tabular}{|c|c|c|c|c|c|c|c|c|c|c|c|c|}
\hline \multirow{2}{*}{ Antimicrobiano } & \multicolumn{3}{|c|}{ C 1} & \multicolumn{3}{|c|}{ C 2} & \multicolumn{3}{|c|}{ C 3} & \multicolumn{3}{|c|}{ C 4} \\
\hline & $P$ & $\mathrm{~N}$ & $\mathrm{~S}$ & $P$ & $\mathrm{~N}$ & $\mathrm{~S}$ & $P$ & $\mathrm{~N}$ & $\mathrm{~S}$ & $\bar{P}$ & $\mathrm{~N}$ & $\mathrm{~S}$ \\
\hline Amoxicilina & 100 & 0 & 0 & 100 & 0 & 0 & 100 & 0 & 0 & 100 & 0 & 0 \\
\hline Ampicilina & 100 & 0 & 0 & 100 & 0 & 0 & 100 & 0 & 0 & 100 & 0 & 0 \\
\hline Cloxacilina & 96,66 & 0 & 3,34 & 100 & 0 & 0 & 100 & 0 & 0 & 100 & 0 & 0 \\
\hline Oxacilina & 100 & 0 & 0 & 100 & 0 & 0 & 96,66 & 0 & 3,34 & 100 & 0 & 0 \\
\hline Penicilina & 100 & 0 & 0 & 100 & 0 & 0 & 100 & 0 & 0 & 93,33 & 0 & 6,67 \\
\hline Oxitetraciclina & 0 & 100 & 0 & 0 & 100 & 0 & 0 & 100 & 0 & 100 & 0 & 0 \\
\hline Tetraciclina & 0 & 100 & 0 & 0 & 96,66 & 3,34 & 0 & 96,66 & 3,34 & 0 & 0 & 100 \\
\hline Eritromicina & 10 & 0 & 90 & 0 & 100 & 0 & 100 & 0 & 0 & 100 & 0 & 0 \\
\hline Tilosina & 0 & 100 & 0 & NR & NR & NR & 100 & 0 & 0 & 100 & 0 & 0 \\
\hline Sulfadiazina & 100 & 0 & 0 & 100 & 0 & 0 & 100 & 0 & 0 & 100 & 0 & 0 \\
\hline Sulfametoxazol & NR & NR & NR & 100 & 0 & 0 & NR & NR & NR & NR & NR & NR \\
\hline Gentamicina & 0 & 100 & 0 & 6,66 & 93,34 & 0 & 6,66 & 93,34 & 0 & 100 & 0 & 0 \\
\hline Trimetoprim & 0 & 30 & 70 & 0 & 96,66 & 3,34 & 100 & 0 & 0 & 100 & 0 & 0 \\
\hline
\end{tabular}

P: positivo; N: negativo; S: suspeito; NR: não realizado.

C 1: metade do limite de detecção declarado pelo fabricante; C 2: limite máximo de resíduos (IN 42, OMS e Codex Alimentarius); C 3: limite de detecção declarado pelo fabricante; C 4: o dobro do limite de detecção declarado pelo fabricante.

Tabela 3. Detecção (\%) de diferentes concentrações (C) de antimicrobianos adicionados em leite pelo o teste Single (COPAN, Itália)

\begin{tabular}{|c|c|c|c|c|c|c|c|c|c|c|c|c|}
\hline \multirow{2}{*}{ Antimicrobiano } & \multicolumn{3}{|c|}{ C 1} & \multicolumn{3}{|c|}{ C 2} & \multicolumn{3}{|c|}{ C 3} & \multicolumn{3}{|c|}{ C 4} \\
\hline & $\mathrm{P}$ & $\mathrm{N}$ & $\mathrm{S}$ & $\mathrm{P}$ & $\mathrm{N}$ & $\mathrm{S}$ & $\mathrm{P}$ & $\mathrm{N}$ & $\mathrm{S}$ & $P$ & $\mathrm{~N}$ & $\mathrm{~S}$ \\
\hline Amoxicilina & 0 & 100 & 0 & 100 & 0 & 0 & 100 & 0 & 0 & 100 & 0 & 0 \\
\hline Ampicilina & 100 & 0 & 0 & 100 & 0 & 0 & 100 & 0 & 0 & 100 & 0 & 0 \\
\hline Cloxacilina & 100 & 0 & 0 & 100 & 0 & 0 & 100 & 0 & 0 & 100 & 0 & 0 \\
\hline Oxacilina & 100 & 0 & 0 & 100 & 0 & 0 & 100 & 0 & 0 & 100 & 0 & 0 \\
\hline Penicilina & 100 & 0 & 0 & 100 & 0 & 0 & 100 & 0 & 0 & 100 & 0 & 0 \\
\hline Oxitetraciclina & 0 & 100 & 0 & 0 & 100 & 0 & 0 & 100 & 0 & 100 & 0 & 0 \\
\hline Tetraciclina & 0 & 100 & 0 & 0 & 0 & 100 & 0 & 0 & 100 & 100 & 0 & 0 \\
\hline Eritromicina & 0 & 100 & 0 & 0 & 100 & 0 & 100 & 0 & 0 & 100 & 0 & 0 \\
\hline Tilosina & 0 & 100 & 0 & NR & NR & NR & 100 & 0 & 0 & 100 & 0 & 0 \\
\hline Sulfadiazina & 0 & 100 & 0 & 100 & 0 & 0 & 100 & 0 & 0 & 100 & 0 & 0 \\
\hline Sulfametoxazol & NR & NR & NR & 100 & 0 & 0 & NR & NR & NR & NR & NR & NR \\
\hline Gentamicina & 0 & 0 & 100 & 100 & 0 & 0 & 100 & 0 & 0 & 100 & 0 & 0 \\
\hline Trimetoprim & 0 & 87,5 & 12,5 & 0 & 87,5 & 12,5 & 100 & 0 & 0 & 100 & 0 & 0 \\
\hline
\end{tabular}

P: positivo; N: negativo; S: suspeito; NR: não realizado.

C 1: metade do limite de detecção declarado pelo fabricante; C 2: limite máximo de resíduos (IN 42, OMS e Codex Alimentarius); C 3: limite de detecção declarado pelo fabricante; C 4: o dobro do limite de detecção declarado pelo fabricante.

Em relação aos antimicrobianos do grupo betalactâmico (amoxicilina, ampicilina, cloxacilina, oxacilina e penicilina), os testes detectaram as concentrações testadas, em praticamente todas as amostras. Os testes foram sensíveis para detectar as concentrações informadas pelo fabricante (C3), as concentrações estabelecidas pelo LMR (C2) e também metade da concentração informada pelo fabricante (C1), exceto o teste Single para amoxicilina, conforme mostrado nas Tab. 2 e 3. Ambos os testes apresentaram elevada 
sensibilidade para os antimicrobianos do grupo betalactâmico, podendo, então, ser indicados como adequados para a detecção desses resíduos.

A deteç̧ão de concentrações abaixo do LMR pelos testes pode ser vantajosa para o consumidor, mas não para os produtores, pois, nas amostras de leite com concentrações destes antimicrobianos permitidas pela legislação, o teste pode apresentar resultado positivo, levando à penalização desse produtor e ao descarte desse leite, quando, na verdade, as concentrações estão dentro dos padrões legais. Esses resultados são considerados falso-violativos, pois o teste detecta a concentração de antimicrobiano existente, mesmo que essa esteja dentro do limite permitido pela legislação, o que não seria uma violação.

Quanto aos macrolídeos (eritromicina e tilosina), os kits detectaram as concentrações informadas pelo fabricante e o dobro desse valor (C 3 e C 4, respectivamente) em $100 \%$ das amostras. Porém, o limite de detecção de eritromicina pelos testes, descrito em seu manual de instrução, é superior ao LMR estabelecido pela legislação $(40 \mu \mathrm{g} / \mathrm{L})$. Para a tilosina não há LMR estabelecido na legislação brasileira, e as organizações internacionais estabelecem valor de $50 \mu \mathrm{g} / \mathrm{L}$ (Pedersen e Suhren, 2000), e os testes Microplate e Single detectaram concentrações superiores $(75 \mu \mathrm{g} / \mathrm{L}$ e $100 \mu \mathrm{g} / \mathrm{L}$, respectivamente). Assim, pode-se observar que os testes detectam concentrações acima do permitido pela legislação, o que pode gerar resultados falsonegativos para essas drogas, situação que não é nada favorável ao consumidor e às indústrias e, assim, os kits não podem ser considerados adequados para a detecção desse grupo de drogas.

Os testes apresentaram elevada sensibilidade $(100 \%)$ para antimicrobianos do grupo das sulfonamidas (sulfadiazina e sulfametoxazol), detectando os limites estabelecidos pela legislação brasileira (C2). Então, pode-se afirmar que os testes Microplate e Single são adequados para a pesquisa de resíduos de sulfonamidas em leite.

Em relação às tetraciclinas (oxitetracilina e tetraciclina), os resultados observados no presente estudo indicam que a sensibilidade dos testes foi baixa nas concentrações 2 e 3, demonstrando dificuldades na utilização de tais métodos para detecção destes antimicrobianos, especificamente. No entanto, analisando-se os limites de detecção revistos pelo fabricante (após a realização deste trabalho), de 250 a $300 \mu \mathrm{g} / \mathrm{L}$ para a oxitetraciclina e de 200 a $300 \mu \mathrm{g} / \mathrm{L}$ para a tetraciclina, verifica-se que os testes realmente não detectam os LMR $(100 \mu \mathrm{g} / \mathrm{L})$, mas concentrações mais elevadas. Este resultado é coerente com o encontrado por Hotta (2003), que, utilizando os mesmos métodos microbiológicos para detecção de resíduos de antimicrobianos em leite cru, não encontrou resultados positivos para detecção de $100 \mu \mathrm{g} / \mathrm{L}$ de tetraciclinas. O fato de os testes Microplate e Single detectarem valores superiores aos estabelecidos pela legislação brasileira para resíduos de tetraciclinas pode gerar resultados falso-negativos. Assim, quando laboratoristas de indústrias de laticínios utilizam esses testes, amostras com resultados negativos podem, na verdade, estar positivas, veiculando resíduos deste antimicrobiano no leite. As consequências da presença de tais resíduos no leite podem ser caracterizadas por problemas tecnológicos para as indústrias e de saúde pública. Diante da análise destes resultados, pode-se indicar que os testes Microplate e Single não são adequados para a detecção de resíduos de tetraciclinas dentro do LMR estabelecido pela legislação brasileira.

Para a gentamicina, o teste Microplate foi capaz de detectar o dobro do LMR estabelecido pelo Codex Alimentarius para essa droga $(200 \mu \mathrm{g} / \mathrm{L})$. Nas amostras analisadas, o teste detectou a concentração do LMR em apenas 6,7\% dos resultados. Entretanto, o limite de detecção informado pelo fabricante após revisão (250 a $350 \mu \mathrm{g} / \mathrm{L}$ ) foi superior ao LMR, e com isso, não se pode afirmar que o teste não está condizente com o manual de instruções, apesar de o teste não ter detectado o LMR. O teste Single foi capaz de detectar o LMR para a gentamicina, portanto sendo mais eficiente que o Microplate na detecção de resíduos desse antimicrobiano em leite, de acordo com os valores estabelecidos pela legislação brasileira.

Na pesquisa de resíduos de trimetoprim, os testes Microplate e Single foram capazes de detectar em $100 \%$ das amostras testadas uma concentração quatro vezes maior que o LMR estabelecido pela Organização Mundial da Saúde $(50 \mu \mathrm{g} / \mathrm{L})$, que é o valor de referência utilizado, 
uma vez que a legislação brasileira não estabelece LMR para esta droga. Embora os kits não tenham detectado os valores do LMR para o trimetoprim, os resultados foram coerentes, visto que os mesmos detectaram os limites estabelecidos no manual do fabricante $(200 \mu \mathrm{g} / \mathrm{L})$. Porém, esses testes não são adequados para a pesquisa desse antimicrobiano em leite por detectar concentrações acima do permitido pela legislação.

Portanto, os resultados obtidos mostraram que os testes COPAN (Microplate e Single) foram eficientes na detecção de antimicrobianos betalactâmicos, sulfametazinas e tilosina, conforme descrito por Gorni e Cabrini (2003), os quais afirmam que os testes microbiológicos, como o Delvotest SP e o COPAN, abrangem as principais classes de antimicrobianos utilizados em gado leiteiro, como os betalactâmicos, as tetraciclinas, as sulfas e os aminoglicosídeos, possuindo excelente limite de detecção para as penicilinas e as sulfas, razoável para as tetraciclinas, questionável para a gentamicina e péssimo para o cloranfenicol.

\section{CONCLUSÕES}

Os testes qualitativos COPAN Microplate e Single mostraram-se eficientes na detecção de antibióticos do grupo betalactâmico apresentando $100 \%$ de sensibilidade na detecção de amoxicilina, ampicilina, cloxaxilina, penicilina, sulfadiazina, sulfametoxazol, tilosina e $96,7 \%$ de oxacilina. Os testes também foram sensíveis a todos os limites de detecção dos antimicrobianos testados, declarados pelo fabricante. Considerando-se os padrões estabelecidos pela legislação brasileira e internacional, verificou-se que os testes COPAN Microplate e Single foram capazes de detectar os LMR para a maioria dos antimicrobianos avaliados: amoxicilina, ampicilina, cloxaxilina, oxacilina, penicilina, sulfadiazina, sulfametoxazol e gentamicina (Single). Entretanto, os testes não se mostraram eficientes na detecção das concentrações estabelecidas pela legislação (LMR) para eritromicina, oxitetraciclina, tetraciclina, trimetoprim e gentamicina (Microplate).

\section{REFERÊNCIAS BIBLIOGRÁFICAS}

BORGES, G.T.; SANTANA, A.P.; MESQUITA, A.J. et al. Ocorrência de resíduos de antibióticos em leite pasteurizado integral e padronizado produzido e comercializado no Estado de Goiás. Cienc. Anim. Bras., v.1, p.59-63, 2000.

BRADY, M.S.; KATZ, S.E. Antibiotic/antimicrobial residues in milk. $J$. Food Prot., v.51, p.8-11, 1988.

BRITO, M.A.V.P. Normas internacionais e exigências do Codex Alimentarius e comparação entre blocos comerciais sobre a adoção de testes para detecção de resíduos de antibióticos no leite. In: BRITO, J.R.F. (Ed). Diagnóstico da qualidade do leite, impacto para a indústria e a questão dos resíduos de antibióticos. Juiz de Fora: Embrapa Gado de Leite, 2003. p.65-76.

BRITO, M.A.V.P. Resíduos de antibióticos no leite: um problema que tem solução. Juiz de Fora: Embrapa Gado de Leite, 2006. Disponível em: $<$ http://www.cnpgl.embrapa.br $>$. Acessado em: 5 set. 2006 .

CARNEIRO, D.O; FIGUEIREDO, H.C.P.; PEREIRA JÚNIOR, D.J. et al. Perfil de susceptibilidade a antimicrobianos de bactérias isoladas em diferentes sistemas de cultivo de tilápia-do-nilo (Oreochromis niloticus). Arq. Bras. Med. Vet. Zootec., v.59, p.869-876, 2007.

CERQUEIRA, M.M.O.P. Detecção de resíduos de antibióticos em leite - Testes disponíveis e considerações. In: BRITO, J.R.F. (Ed). Diagnóstico da qualidade do leite, impacto para a indústria e a questão dos resíduos de antibióticos. Juiz de Fora: Embrapa Gado de Leite, 2003. p.77-87.

COSTA, E.O. Resíduos de antibióticos no leite: um risco à saúde do consumidor. Rev. Hig. Alim., v.10, p.15-17, 1996.

EURACHEM guide: the fitness for purpose of analytical methods. Teddington, UK: Eurachem, LGC, 1998. 61p.

GORNI, R.; CABRINI, S. Dificuldades e demandas da indústria sobre testes para detecção de resíduos de antibióticos em leite. In: BRITO, J.R.F. (Ed). Diagnóstico da qualidade do leite, impacto para a indústria e a questão dos resíduos de antibióticos. Juiz de Fora: Embrapa Gado de Leite, 2003. p.97-102.

HOTTA, J.M. Monitoramento de resíduos de antimicrobianos em diferentes pontos da cadeia produtiva do leite, comparando diferentes métodos de detecção. 2003. 90f. Dissertação 
(Mestrado) - Escola de Veterinária, Universidade Federal de Minas Gerais, Belo Horizonte.

INSTRUÇÃO Normativa $\mathrm{n}^{\circ} 42$, de 20 de dezembro de 1999, que altera o Plano Nacional de Controle de Resíduos em Produtos de Origem Animal - PNCR. [do Ministério da Agricultura Pecuária e Abastecimento, Brasília, DF]. Diário Oficial da União: Brasília, em 22 de dezembro de 1999. Seção 1, página 13. Disponível em: $<$ http://www.agricultura.gov.br $>$. Acessado em: 10 abr. 2005.

JOHNSSON, G. Swedish scheme for the control of inhibitory substances. Bull. Int. Dairy Fed., n.283, p.59-60, 1993.

MACHADO, T.R.O.; CORREA, M.G.; MARIN, J.M. Antimicrobial susceptibility of coagulasenegative Staphylococci isolated from mastitic cattle in Brazil. Arq. Bras. Med. Vet. Zootec., v.60, p.278-282, 2008.

MITCHEL, J.M.; GRIFFITHS, M.W.; McEWEN, S.A. et al. Antimicrobial drug residues in milk and meat: causes, concerns, prevalence, regulations, tests, and tests performance. J. Food Prot., v.61, p.742-756, 1998.

MOREIRA, M.A.S.; MORAES, C.A. Resistência a antibióticos em bactérias Gramnegativas isoladas de carcaças de frangos. Arq. Bras. Med. Vet. Zootec., v.54, p.1-7, 2002.

NERO, L.A.; MATTOS, M.R.; BELOTI, V. et al. Hazards in non-pasteurized milk on retail sale in Brazil: prevalence of Salmonella spp., Listeria monocytogenes and chemical residues. Braz. J. Microbiol., v.35, p.211-215, 2004.

PEDERSEN, M.; SUHREN, G. Chemicalphysical confirmation tests ("higher" validation levels) for the detection of residues of antimicrobials in milk. Bull. Int. Dairy Fed., n.358, p.29-35, 2000.

PORTARIA n51, de 18 de setembro de 2002 , que aprova os Regulamentos Técnicos de Produção, Identidade e Qualidade do Leite tipo A, do Leite tipo B, do Leite tipo C, do Leite Pasteurizado e do Leite Cru Refrigerado e o regulamento Técnico da Coleta de Leite Cru Refrigerado e seu Transporte a Granel [do Ministério da Agricultura Pecuária e Abastecimento, Brasília, DF]. Diário Oficial da União: Brasília, em 20 de setembro de 2002. Seção 1, página 13. Disponível em: $<$ http://www.agricultura.gov.br $>$. Acessado em: 10 abr. 2005.

RAMÍREZ, A.; GUTIÉRREZ, R.; GONZÁLEZ, C. et al. Detección de antibióticos en leche comercializada en la ciudad de México. Rev. Salud Anim., v.23, p.37-41, 2001.

SCHLIEPHAKE, A. A comparative study of a newly developed agar-diffusion test and the brilliant-black reduction tests in conjunction with an ELISA-reader to measure antibiotic residues in milk. Milchwissenschaft, v.53, p.88-90, 1998. 